\title{
A Review of Attention Bias and Eating Disorder
}

\author{
Lijing OUYANG ${ }^{1, *}, \dagger$ Yingqi $\mathrm{WU}^{2, *, \dagger}$
}

\author{
${ }^{1}$ Brentwood School, Brentwood, Essex, UK \\ +447577698236/+8618515194576 \\ *1185562152@qq.com \\ ${ }^{2}$ Guangdong Country Garden School, Foshan, Guangdong, China \\ $+8613418405450$ \\ *820494707@qq.com \\ These authors contributed equally.
}

\begin{abstract}
Eating disorder (ED) is a mental disorder defined by abnormal eating habits that negatively affect a person's physical and psychological health. Attentional bias $(\mathrm{AB})$ is a kind of cognitive bias, refers to the differential assignment of attention to special stimuli and involves the method of selective attention. It is essential to investigate cognitive explanations like the AB of EDs, as this can allow us to know how people think and how their thoughts have linked to food choices and eating habits, thereby reducing the number of people being eating disordered by changing their ways of thinking via appropriate treatments. In this paper the $\mathrm{AB}$ as a possible cognitive explanation of EDs has been discussed. We found that Individuals with EDs are more sensitive to food cues, especially those high-calories, which is supported by results of using eye tracking and electroencephalo-graph. Disadvantageous social comparisons and impaired social functions might contribute to ED. In terms of EDs treatment, cognitive behavioral therapy, like Attention bias modification has been utilized and become an efficacious intervention.
\end{abstract}

Keywords: eating disorder, attentional bias, experiments evidence, future treatment.

\section{INTRODUCTION}

In psychology, an eating disorder is a mental disorder defined by abnormal eating habits that negatively affect a person's physical and psychological health. It is crucial to study eating disorders as they have become a global epidemic in the last few decades, and why this has occurred needs to be investigated. It is essential to investigate cognitive explanations like the attentional bias $(\mathrm{AB})$ of eating disorders, as this can allow us to know how people think and how their thoughts have linked to food choices and eating habits, and therefore reduce the number of people being eating disordered by changing their ways of thinking via appropriate treatments. Feeding and eating disorders are characterized by a persistent disturbance of eating or eating-related behavior resulting in the altered consumption or absorption of food, which significantly impairs physical health or psychological functioning [1]. The cause of these disorders is presumed to be complex and multiply influenced by developmental, social, and biological processes [2]. Its classification is classified into anorexia nervosa, bulimia nervosa, binge eating disorder, and other specified feeding or eating disorders according to ICD-10 and the DSM-5 [1]. This essay will discuss $\mathrm{AB}$ as a possible cognitive explanation of eating disorders.

$\mathrm{AB}$ is a kind of cognitive bias, it refers to the differential assignment of attention to special stimuli and involves the method of selective attention [4]. In some cases, people's attention becomes focused on just a few of the votes while ignoring the rest. $\mathrm{AB}$ refers to how a person's perception is affected by selective factors in their attention. ABs may explain an individual's failure to consider alternative possibilities when occupied with an existing series of thoughts. For example, cigarette smokers have been shown to possess an $\mathrm{AB}$ for smokingrelated cues around them due to their brain's altered reward sensitivity [4]. Obese people may only focus on the food they usually have, which is often unhealthy because they have already got a habit. AB has also been associated with clinically relevant symptoms such as anxiety and depression; it can also impact decisionmaking and addictive behaviors. 
Several literature searches have proposed the role of risk factor in temperament in eating disorders, while a comprehensive theory about how $\mathrm{AB}$ influences eating disorders (EDs) has not been proposed. For example, a cognitive model suggests individuals with EDs are oversensitive to body shape caused by environmental pressures, which could be a maintenance factor [5]. Another research paper also has concluded that the function of attention biases in ED is that the dissatisfaction of body parts leads to extreme dietary restriction to improve self-image [6]. Therefore, the attention bias over concerns to body shape and body parts may finally lead to people have ED symptoms. This work reviews how $\mathrm{AB}$ perpetuates the maintenance of $\mathrm{ED}$ symptoms and potential treatment. First, we will review some experiment evidence regarding the AB's influence on $\mathrm{BD}$ in individuals. Next, some useful treatments relevant to $\mathrm{AB}$ for $\mathrm{BD}$ will be presented.

\section{EXPERIMENTAL EVIDENCES}

\subsection{Eye-Tracking experiments}

Individuals with EDs have been shown to possess an $\mathrm{AB}$ for disorder-related cues [7]. Eye-tracking has been utilized in the further exploration of the relationship between $\mathrm{ABs}$ and EDs. It tracks eye gaze, revealing attention allocation for cues, thus providing clear perception and attention processes [7]. The first study investigates the engagement and maintenance of ABs to food cues in adults with binge ED [8]. When high-calorie and low-calorie food items are presented in the same images, and other different images are shown at the same time, adults with binge ED have a longer duration for those food-related images than the controls [8]. The second study shows that all participants allocate more attention to non-food stimuli than food stimuli in the free exploration task.

In contrast, the result of eye gaze tracking shows a prolonged duration in those with binge ED compared to the weight-matched controls [9]. During the visual search task, a shorter time is required in detecting food targets for participants with full-syndrome binge ED than controls [9]. The research findings support the idea that $\mathrm{ABs}$ to food-related cues for individuals with binge ED [9].

Additionally, a study has examined the gaze patterns and body satisfaction of adolescents with EDs [10]. Participants with EDs and healthy controls both exhibit attentional engagement to unattractive areas of one's body; Girls with anorexia nervosa demonstrate notable prolong in the duration to unsightly body areas but shorten the time for attractive body areas than the controls [10]. All subgroups of EDs state significant dissatisfaction with their body shape. However, this score is remarkably lower in binge ED than that of anorexia nervosa, indicating that the stronger the deficit orientation to the body, the more significant the body dissatisfaction [10]. Finally, a study in females illustrates a drop in body satisfaction in exposure to media content pursuing thinner body shape [11]. This tendency is more apparent in patients with bulimia nervosa, because the recorded gaze pattern suggests that they have a more extended fixation on the lower BMI body images than the controls, whereas the way reverses for higher BMI bodies [11]. Upward social comparison plays a crucial role in increasing body dissatisfaction in bulimia Nervosa, resulting in ED maintenance [11].

In conclusion, eye gaze tracking suggests prolonged engagement and $\mathrm{ABs}$ to food cues in individuals with EDs. There is evidence that those people have increased body dissatisfaction, especially exposure to disadvantageous social comparisons.

\subsection{EEG experiments}

Six studies have assessed the attention processing with event-related potentials (ERPs) of participants with binge eating behaviors. Four of them examined $\mathrm{AB}$ to delicious food cues, one used weight or shape signals, and two used threat cues.

One of the studies was carried out by Blechert et al. in 2011. In this study, they compared the electrocortical processing of food pictures in participants with either anorexia nervosa or bulimia nervosa, and healthy controls by measuring the early posterior negativity, which is an event-related potential that reflects selective attention and stimulus salience [12]. They exposed the three groups to a rapid stream of high-calorie and lowcalorie food pictures, as well as standard emotional and neutral views [12]. The result shows that in the time range of 220 milliseconds to 310 milliseconds, the posterior electrodes' ERPs differed between groups. The patients with EDs showed facilitated processing in both highcalorie and low-calorie food pictures related to neutral images, while with healthy control participants this only happened for the high-calorie pictures. The subjective palatability rate of the pictures was shown in this order: patients with anorexia nervosa the highest, followed by the healthy control group, then bulimia nervosa groups [12]. According to the study, women with bulimia nervosa showed greater attentional engagement toward all food images and non-food images [13]. On the other hand, women without bulimia nervosa showed greater attentional engagement toward high-calorie food versus low-calorie food and non-food ideas [13]. In conclusion, patients with EDs display a generalized $\mathrm{AB}$ for food images, no matter the caloric value, which may explain the persistent preoccupation with food in these individuals.

Kuhnpast, Gramann, and Pollatos carried out another study in 2012. This study used threat cues, in which individuals viewed images of male and female faces with 
angry, fearful, happy, and neutral expressions [14]. The study aims to investigate electrophysiologic evidence for deficits in emotional face processing in patients with bulimia nervosa [14]. ERPs were noted from 13 women with bulimia nervosa, and 13 matched healthy controls when viewing neutral, happy, fearful, and angry facial expressions. Participants' recognition performance for emotional faces was tested in a subsequent categorization task. The degree of alexithymia, depression and anxiety were also assessed via questionnaires [14]. The result showed that categorization of emotional faces was hampered in bulimia nervosa, amplitudes of ERPs varied during emotional face processing: face-specific N170 amplitudes were less pronounced for angry faces in patients with bulimia nervosa [14]. Women with bulimia nervosa had more difficulties categorizing angry faces in the early presentation intervals than controls, showing emotion recognition deficits at the facilitated attentional engagement stage [13]. Women with bulimia nervosa showed more significant attentional disengagement difficulties across all face types relative to controls [13]. In conclusion, the study provides novel electrophysiologic data showing that emotional faces are processed differently in patients with $\mathrm{BN}$ compared to healthy controls, and these findings might contribute to a better understanding of the impaired social functioning in BN [14].

Thinking altogether, EEG studies using food cues give us mixed evidence for facilitated attentional engagement and disengagement difficulties in women with binge eating behaviors.

\section{TREATMENTS}

\subsection{Utilizing attention bias modification in eating disorder psychopathology}

Attention bias modification (ABM) is developed to control anxiety by reducing attention bias to threats originally [15]. Two studies have been done that state the effect of utilizing $\mathrm{ABM}$ in treating $\mathrm{AB}$. The dot-probe paradigm is the most used ABM intervention [15]. Patients are instructed to identify the probe appearing on substance-relevant or substance-irrelevant stimuli by press the key [16]. In the training session, this probe always appears with substance-irrelevant incentives, and thus patients shift their attention from threat-related stimuli [16]. A study examines the effect of visual search $\mathrm{ABM}$ in adolescents suffering from anxiety and depression. Researchers assessed $\mathrm{AB}$ and stressreactivity before and after the experiment. The results suggest that a reduction is generated in $\mathrm{AB}$ in the visual search $\mathrm{ABM}$ training group compared to a placebo group and the controls [17]. Additionally, a proportional relationship has been shown between training amounts and reduction in $\mathrm{AB}$ [17]. To sum up, $\mathrm{ABM}$ serves as a positive guideline in treating $\mathrm{AB}$.
Based on previous statements and research, even though the interaction between $\mathrm{AB}$ and $\mathrm{ED}$ could be identified, extinction appetitive response is more significant and challenging [17]. One study conducted in the binge ED patients assesses and modifies food-related biases, including the fluctuation and duration of the biases [17]. Participants were exposed to either enhancement or reduction group randomly [17]. This experiment shows a successful reduction in the foodrelated $\mathrm{AB}$ in the reduction group while the effect persists briefly [17]. Moreover, compared to a decrease in AB, the possible increase in bias is less significant [17]. Another study was carried out in 56 patients with binge ED or bulimia nervosa, and provided a similar result [18]. Participants got involved in real ABM that participants were trained to show avoidance behavior in response to food cues or sham ABM without such training [18].

Consequently, symptoms of ED, food craving, and food reactivity are reduced in both groups, and this reduction is greater in real ABM [18]. Besides, a study investigated whether ABM can inoculate people against appetitive food cues, for instance, an advertisement for chocolate products [19]. Undergraduate women were trained by a modified dot-probe task to direct their attention toward or away chocolate products and the taste test was a measure of chocolate consumption [19]. The result states that there is an obvious reduction in $\mathrm{AB}$ for chocolate in subjects who underwent avoid condition while not that significant in chocolate consumption, meaning that the inoculation effect is solely confided to $\mathrm{AB}$ regardless of consumption [19]. Taken together, massive researches support that the efficacy of ABM in treating the $\mathrm{ED}$, and some view $\mathrm{ABM}$ as a potentially efficacious intervention due to its dramatic impact on reducing $\mathrm{AB}[20]$.

\subsection{Future directions}

It seems reasonable that $\mathrm{ABM}$ may help with treating ED symptoms, according to the strength of the previous findings on $\mathrm{AB}$ in $\mathrm{EDs}$, and the data collected from the few attention-based ED studies [20]. However, one of the major difficulties in effectively treating EDs is resistance. For example, as $\mathrm{AB}$ serves the perceived role of protecting the patient from the feared outcome of weight gain, low self-worth and ultimately social rejection, many patients with EDs have huge difficulty letting go of their ABs, such as body/weight checking [20]. Treating these biases using ABM is very important because it will allow us to treat patients suffering from biases more accurately and efficiently [21]. We found one study on this topic that concluded $A B$ in a sample of female participants with clinical EDs decreased after a successful course of CBT (cognitive behavioral therapy) [20]. In the study, biases were found for negative eating and neutral weight pictures and positive eating pictures in women with EDs. These biases were more significant 
than those in anxious and normal controls [20]. It is concluded that future research should establish whether such tendencies warrant specific treatment [20]. In the future, we also need to consider if the results of the study can be applied to males as the participants were females, as well as the continuation of the effect.

\section{CONCLUSION}

In summary, this paper argues to the possible maintenance factors of $\mathrm{AB}$. Individuals with EDs are more sensitive to food cues, especially those highcalories, which is supported by results of eye tracking and EEG. Disadvantageous social comparisons and impaired social functions might contribute to ED. In terms of EDs treatment, CBT, like ABM, has been utilized and become an efficacious intervention. However, the major difficulties in treating EDs are resistance from patients, resulting from incorrect understanding about the disorder. Overall, this work can provide well-rounded knowledge for researchers, which incorporates influencing factors and potential intervention of the EDs. It can be concluded that further research is needed to confirm the causes and specific treatment for EDs.

\section{REFERENCES}

[1] American Psychiatric Association (2013). Diagnostic and Statistical Manual of Mental Disorders (5th edition). Arlington, VA: American Psychiatric Association.

[2] Klump, K. L., Kaye, W. H., \& Strober, M. (2001). The evolving genetic foundations of eating disorders. Psychiatric Clinics of North America, 24(2), 215-225.

[3] Drobes, D. J., Elibero, A., \& Evans, D. E. (2006). Attentional bias for smoking and affective stimuli: A Stroop task study. Psychology of addictive behaviors, 20(4), 490.

[4] Cisler, J. M., \& Koster, E. H. (2010). Mechanisms of attentional biases towards threat in anxiety disorders: An integrative review. Clinical psychology review, 30(2), 203-216.

[5] Vitousek, K. B., \& Hollon, S. D. (1990). The investigation of schematic content and processing in eating disorders. Cognitive therapy and research, 14(2), 191-214.

[6] Aspen, V., Darcy, A. M., \& Lock, J. (2013). A review of attention biases in women with eating disorders. Cognition \& emotion, 27(5), 820-838.

[7] Kerr-Gaffney, J., Harrison, A., \& Tchanturia, K. (2019). Eye-tracking research in eating disorders: A systematic review. International Journal of Eating Disorders, 52(1), 3-27.
[8] Popien, A., Frayn, M., von Ranson, K. M., \& Sears, C. R. (2015). Eye gaze tracking reveals heightened attention to food in adults with binge eating when viewing images of real-world scenes. Appetite, 91, 233-240

[9] Sperling, I., Baldofski, S., Lüthold, P., \& Hilbert, A. (2017). Cognitive food processing in binge-eating disorder: an eye-tracking study. Nutrients, 9(8), 903.

[10] Bauer, A., Schneider, S., Waldorf, M., Braks, K., Huber, T. J., Adolph, D., \& Vocks, S. (2017). Selective visual attention towards oneself and associated state body satisfaction: an eye-tracking study in adolescents with different types of eating disorders. Journal of Abnormal Child Psychology, 45(8), 1647-1661.

[11] Blechert, J., Nickert, T., Caffier, D., \& TuschenCaffier, B. (2009). Social comparison and its relation to body dissatisfaction in bulimia nervosa: Evidence from eye movements. Psychosomatic Medicine, 71(8), 907-912.

[12] Blechert, J., Feige, B., Joos, A., Zeeck, A., \& Tuschen-Caffier, B. (2011). Electrocortical processing of food and emotional pictures in anorexia nervosa and bulimia nervosa. Psychosomatic Medicine, 73(5), 415-421.

[13] Stojek, M., Shank, L. M., Vannucci, A., Bongiorno, D. M., Nelson, E. E., Waters, A. J., Engel, S. G., Boutelle, K. N., Pine, D. S., Yanovski, J. A., \& Tanofsky-Kraff, M. (2018). A systematic review of attentional biases in disorders involving binge eating. Appetite, 123, 367-389.

[14] Kühnpast, N., Gramann, K., \& Pollatos, O. (2012). Electrophysiologic evidence for multilevel deficits in emotional face processing in patients with bulimia nervosa. Psychosomatic Medicine, 74(7), 736-744.

[15] Mogg, K., Waters, A. M., \& Bradley, B. P. (2017). Attention bias modification (ABM): Review of effects of multisession ABM training on anxiety and threat-related attention in high-anxious individuals. Clinical Psychological Science, 5(4), 698-717.

[16] MacLeod, C., Rutherford, E., Campbell, L., Ebsworthy, G., \& Holker, L. (2002). Selective attention and emotional vulnerability: assessing the causal basis of their association through the experimental manipulation of attentional bias. Journal of abnormal psychology, 111(1), 107.

[17] De Voogd, E. L., Wiers, R. W., \& Salemink, E. (2017). Online visual search attentional bias modification for adolescents with heightened anxiety and depressive symptoms: A randomized 
controlled trial. Behaviour Research and Therapy, 92, 57-67.

[18] Brockmeyer, T., Friederich, H. C., Küppers, C., Chowdhury, S., Harms, L., Simmonds, J., ... \& Schmidt, U. (2019). Approach bias modification training in Bulimia nervosa and binge-eating disorder: a pilot randomized controlled trial. International Journal of Eating Disorders, 52(5), 520-529.

[19] Kemps, E., Tiggemann, M., \& Stewart-Davis, E. (2018). Can attentional bias modification inoculate people to withstand exposure to real-world food cues?. Appetite, 120, 222-229.

[20] Shafran, R., Lee, M., Cooper, Z., Palmer, R. L., \& Fairburn, C. G. (2007). Attentional bias in eating disorders. The International journal of eating disorders, 40(4), 369-380.

[21] Aspen, V., Darcy, A. M., \& Lock, J. (2013). A review of attention biases in women with eating disorders. Cognition \& emotion, 27(5), 820-838. 